\title{
SUSI's Potential for Double Star Research
}

\author{
JOHN DAVIS \\ Chatterton Astronomy Department, Institute of Astronomy, \\ School of Physics, University of Sydney
}

\begin{abstract}
The Sydney University Stellar Interferometer (SUSI) is currently undergoing commissioning and will soon commence its astronomical program in which observations of double stars will form a major component. With its 640-m long NorthSouth array of input siderostats, the new instrument will have unprecedented angular resolution.
\end{abstract}

\section{INTRODUCTION}

The purpose of this brief contribution is to alert the double and multiple star community to the fact that an instrument of unprecedented angular resolution will soon commence astronomical observations. The Sydney University Stellar Interferometer, or SUSI as she has become known, has been developed by the Chatterton Astronomy Department as a successor to the Narrabri Stellar Intensity Interferometer (NSII) (Hanbury Brown et al. 1967). In the context of this meeting it is appropriate to note that the Narrabri instrument was used in the first demonstration of the power of combining interferometry and spectroscopy in the study of double-line spectroscopic binaries (Herbison-Evans et al. 1971).

The new instrument is located at the Paul Wild Observatory of the Commonwealth Scientific and Industrial Research Organisation (CSIRO), roughly $450 \mathrm{~km}$ north of Sydney and west of the Great Dividing Range, in northern New South Wales. SUSI's design is based on a successful $11.4-\mathrm{m}$ baseline prototype interferometer which was used to measure the angular diameter of Sirius (Davis \& Tango 1986). Construction began in late 1987 and currently the inner baselines are being commissioned with science observations to commence shortly. Figure 1 shows an aerial view of SUSI.

It is planned to use SUSI in a wide range of programs in stellar astrophysics, and the study of binary stars will form a major component of the overall program.

\section{THE INSTRUMENT}

On the basis of a study of the potential stellar programs, SUSI has been designed to be at least 100 times more sensitive than the NSII which had a limiting magnitude of $B=+2.5$. An outline of the design of SUSI has been given by Davis \& Tango (1985b). Unlike the NSII, which was inherently limited to stars of spectral type earlier than the Sun, SUSI will be capable of measuring stars of all spectral types brighter than its predicted limiting magnitude of about +8 .

A very brief description of SUSI is that it is an optical, two-aperture, single $r_{0}$, amplitude interferometer with wavefront-tilt correction and fringe tracking. It has a North-South array of input stations giving baselines increasing in approximately geometric progression from $5 \mathrm{~m}$ to $640 \mathrm{~m}$. The instrumental 


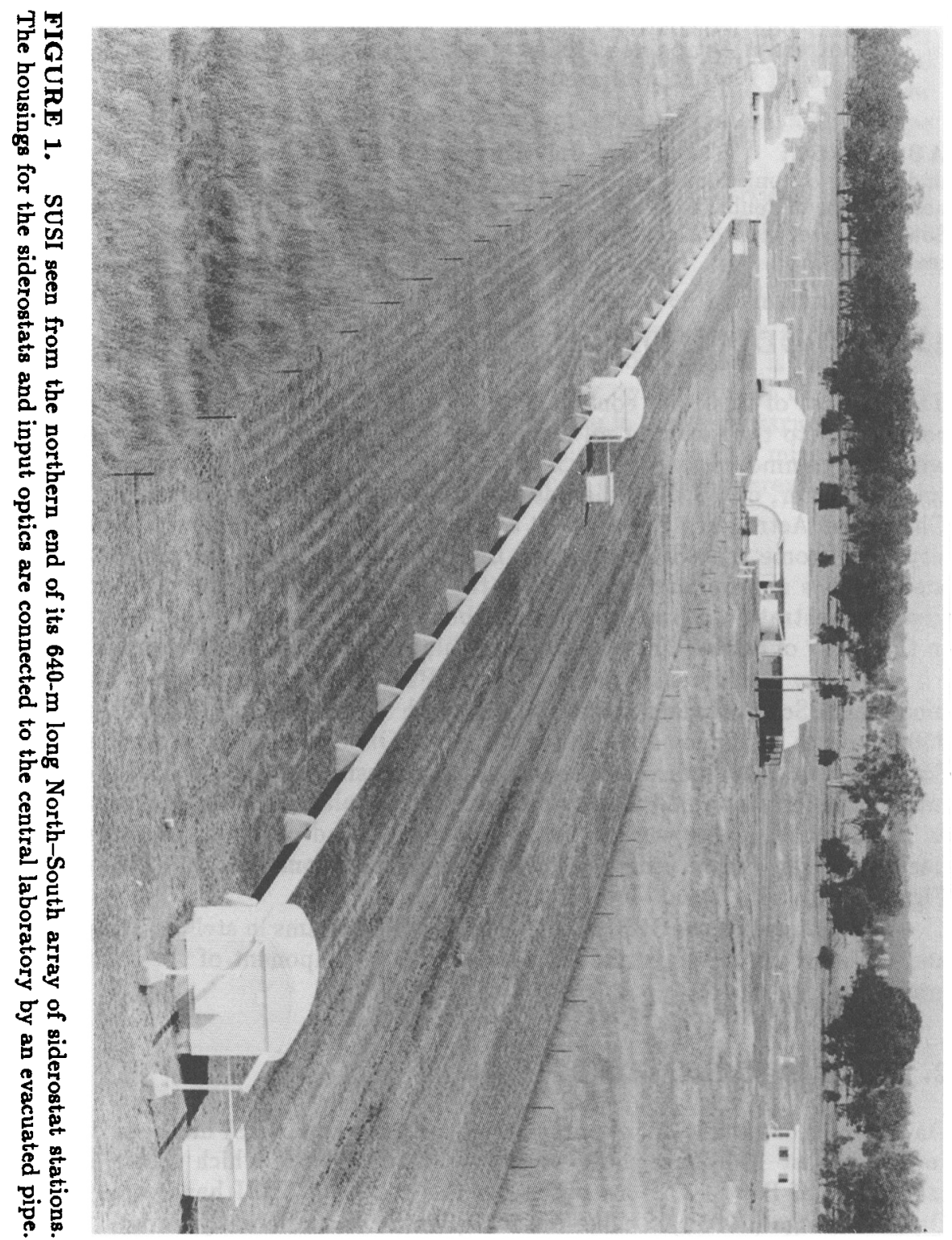


parameters of SUSI are listed in Table 1. SUSI has significant advantages compared with the NSII and Table 2 contains a comparison between the instruments.

TABLE 1. SUSI Parameters

\begin{tabular}{|ll|}
\hline \multicolumn{1}{|c|}{ Parameter } & \multicolumn{1}{c|}{ Value } \\
\hline Baselines & $5 \mathrm{~m}$ to $640 \mathrm{~m}$ North-South \\
Baseline ratio & $\sim 1.4^{\text {a }}$ \\
Angular resolution & $0^{\prime \prime} .02$ to $0^{\prime \prime} .00005$ \\
Max. aperture diameter & $0.14 \mathrm{~m}$ \\
Spectral range & $400-900 \mathrm{~nm}$ \\
Limiting magnitude & $\mathrm{V} \sim+8$ \\
\hline
\end{tabular}

- Every second baseline is longer by a factor of 2 ; intermediate baselines are longer by approximately $\sqrt{2}$.

TABLE 2. The Advantages of SUSI over the NSII

\begin{tabular}{|lcc|}
\hline \multicolumn{1}{|c}{ Property } & SUSI & NSII \\
\hline Limiting magnitude-Single stars & $\sim+8.0$ & +2.5 \\
Limiting magnitude-Double stars & $\sim \sim+6.5$ & +1.0 \\
Angular resolution (mas) & $<1 \times 10^{-4}$ & $3 \times 10^{-4}$ \\
Range in spectral types & 0 to M & O to F \\
Spectral range & $400-900 \mathrm{~nm}$ & $443-465 \mathrm{~nm}$ \\
Spectral resolution $^{b}$ & $0.1 \mathrm{~nm}$ & $2.5-8 \mathrm{~nm}$ \\
Aperture diameter $^{b}$ & $0.14 \mathrm{~m}$ & $6.7 \mathrm{~m}$ \\
\hline
\end{tabular}

a Experience with the NSII showed that the limiting magnitude for binary atars is $\sim 1.5$ magnitudes brighter than for single stars.

- Unlike the NSII, SUSI has no significant partial resolution effect by the aperture diameter.

\section{SUSI'S PROGRAM}

Table 3 summarises some of the programs that will be undertaken with SUSI. It can be seen that studies of binary stars form a major part of the overall program. The technique of observing binary stars with long baseline optical interferometers has been discussed by Davis (1983), and the importance of interferometric observations has been discussed by McAlister $(1979,1985)$ and re-emphasized by both Griffin and McAlister in their contributions to this meeting.

Double-lined spectroscopic binaries are of particular interest for interferometric studies because the ability of a high angular resolution interferometer to measure the orbital inclination, the angular major semi-axis and the angular diameter of the primary of the system enables the distance to the system, the masses of both components, and the radius and luminosity of at least the 
primary component to be determined. Single-lined spectroscopic binaries require the distance to be known independently in order to determine the masses of the components and the radius and luminosity of at least the primary. For eclipsing binaries, interferometry can enable the distance and luminosities of the components to be determined.

TABLE 3. Some Stellar Programs for SUSI

\begin{tabular}{|ll|}
\hline Major Program & \multicolumn{1}{c|}{ Sub-Programs } \\
\hline - Single Stars & o surface fluxes and effective temperatures \\
& $\circ$ radii and luminosities for stars of known parallax \\
& $\circ$ stellar atmospheres \\
& $\circ$ emission line stars \\
& (e.g., Be, shell and Wolf-Rayet stars) \\
& $\circ$ stellar rotation \\
& $\circ$ limb darkening \\
& $\circ$ double-line spectroscopic binaries--distances, \\
& masses, radii, luminosities \\
& $\circ$ single-line spectroscopic binaries of known \\
& parallax-masses, radii, luminosities \\
& $\circ$ eclipsing binaries-distances, luminosities \\
& $\circ$ discovery of previously unknown binary systems \\
& $\circ$ distances, radii, temperatures etc. for \\
& Cepheid and Mira variables \\
\hline
\end{tabular}

The range of angular resolution provided by SUSI will enable a large sample of binary systems to be resolved that cannot be resolved by visual or speckle interferometric techniques. It will provide essentially complete overlap with spectroscopic studies, thus removing the problem of non-overlap of techniques that has handicapped studies of binary stars in the past. For example, apart from $\alpha$ Virginis which was studied with the NSII (Herbison-Evans et al. 1971), there are to my knowledge no systems with periods shorter than about 35 days that have been spatially resolved. SUSI is capable of studying binary systems south of declination $+20^{\circ}$. Considering only the 50 brightest double-lined spectroscopic binaries in the Eighth Catalogue of the Orbital Elements of Spectroscopic Binary Systems (Batten et al. 1989) accessible to SUSI, the periods cover the range from 0.8 to 100 days.

It should be noted that the number of known spectroscopic binaries accessible to SUSI is smaller than that accessible to an instrument at an equivalent northern latitude by a factor of two or more. There is the need for the discovery and spectroscopic study of binary stars in the Southern Hemisphere, particularly double-lined systems, if the full potential of SUSI for binary star studies is to be realised. 


\section{THE CURRENT STATUS}

The inner two siderostats, giving a baseline of $5 \mathrm{~m}$, have been fully commissioned. Pointing solutions have been established, and they have been used to commission the sub-systems common to all baselines. This includes the stellar acquisition system, the wavefront-tilt correction system and the optical path length compensation system (partially). The beam-combining optical system from the prototype interferometer (Davis \& Tango 1985a) has been installed and re-commissioned. The single spectral channel detection system from the prototype is being used for the commissioning tests to determine baseline vector solutions. It will also be used in the first science observations. While tuning of the instrument is continuing, it is currently performing above expectations in terms of raw fringe visibility measurements.

A further three siderostats have been installed and are currently being commissioned to give baselines in the range 5 to $80 \mathrm{~m}$. Another siderostat is ready for installation, and this will complete the series of baselines from 5 to $80 \mathrm{~m}$. The remaining five stations, giving baselines to $640 \mathrm{~m}$, will be equipped with siderostats and relay optics early in 1993, and they will be commissioned progressively. During 1992-93 we also plan to progressively install an atmospheric refraction correction system, a longitudinal dispersion correction system and a PAPA camera for automatic fringe detection and tracking. The current beamcombining optical system is restricted to the blue region of the spectrum (400$500 \mathrm{~nm}$ ) by the multi-layer dielectric coatings used for the beamsplitters. A beam-combining optical system for longer wavelengths is planned for 1994 .

\section{SUMMARY}

SUSI will commence astronomical observations shortly with its inner baselines. Double stars will be a major part of its program and, in particular, double-lined systems from the Eighth Catalogue of the Orbital Elements of Spectroscopic Binary Systems will be given high priority. The need for spectroscopic studies of binary systems south of declination $+20^{\circ}$ is noted.

\section{ACKNOWLEDGMENTS}

The development of SUSI has been shared with W. J. Tango, A. J. Booth, R. A. Minard and R. R. Shobbrook. Significant contributions have been made to technical aspects by S. M. Owens, H. Bennis, M. Paterson and J. Pasiut. Postgraduate students, particularly T. ten Brummelaar, Y. Gilliand, M. Hrynevych, P. Lawson and E. B. Seneta have also made significant contributions. The project has benefitted enormously from the support and contributions made by the CSIRO. The SUSI program is funded jointly by the Australian Research Council and the University of Sydney. The support of the Pollock Memorial Fund and the Science Foundation for Physics within the University of Sydney is also gratefully acknowledged. 


\section{REFERENCES}

Batten, A.H., Fletcher, J.M., \& MacCarthy, D.G. 1989, Eighth Catalogue of the Orbital Elements of Spectroscopic Binary Systems, Pub. Dominion Astrophysical Obs., Victoria, B.C., Vol. XVII

Davis, J. 1983, in Current Technigues in Double and Multiple Star Research, IAU Colloquium No. 62, ed. R.S. Harrington and O.G. Franz, Lowell Obs. Bulletin No. 167, Vol. IX, 191

Davis, J., \& Tango, W.J. 1985a, Proc. Astron. Soc. Australia, 6, 34

Davis, J., \& Tango, W.J. 1985b, Proc. Astron. Soc. Australia, 6, 38

Davis, J., \& Tango, W.J. 1986, Nature, 323, 234

Hanbury Brown, R., Davis, J., \& Allen, L.R. 1967, MNRAS, 137, 375

Herbison-Evans, D., Hanbury Brown, R., Davis, J., \& Allen, L.R. 1971, MNRAS, 151, 161

McAlister, H.A. 1979, in High Angular Resolution Stellar Interferometry, IAU Colla quium No. 50, ed. J. Davis and W.J. Tango, (Chatterton Astronomy Department, University of Sydney), 3-1

McAlister, H.A. 1985, ARA\&A, 23, 59

\section{DISCUSSION}

POPPER: You didn't describe for us the successful test carried out with your prototype instrument, demonstrating the feasibility of the different components of your system.

DAVIS: Time did not allow me to describe the $11.4-\mathrm{m}$ prototype interferometer that we built and used to test many of the sub-systems that have since been incorporated into SUSI. SUSI's design has been based on the experience gained with the prototype which was used successfully to measure the angular size of Sirius. The printed version of my presentation will contain more information and references to the prototype design and to the Sirius measurement.

BALEGA: As I understand, you still need money to finish the project. What will be the total cost of the instrument?

DAVIS: The capital cost of the instrument was approximately US $\$ 2.25$ million, and this has been funded. Currently, we are short on annual funding which is required to complete the commissioning program and to operate the instrument. I am hopeful that this situation will be rectified for 1993 onwards.

WHITE: For every 100 photons entering your instrument, how many are usefully recorded at the back of your instrument?

DAVIS: Currently, the detected photon rate is only of the order of $2 \%$. However, we have had a significant problem with our reflecting components. There appears to have been a chemical reaction between the substrates and the silver/dielectric coatings which has seriously impaired the reflecting efficiency. The problem is in hand, and we hope to improve this significantly to $\sim 5 \%$. 